\title{
BEST MEAN APPROXIMATION TO A 2-DIMENSIONAL KERNEL BY TENSOR PRODUCTS ${ }^{1}$
}

\author{
BY C. A. MICCHELLI AND A. PINKUS
}

Communicated by Walter Gautschi, December 14, 1976

We are concerned with the problem

$$
\min _{u_{i}, v_{i}} \int_{0}^{1} \int_{0}^{1}\left|K(x, y)-\sum_{i=1}^{n} u_{i}(x) v_{i}(y)\right| d x d y,
$$

where $u_{i}, v_{i} \in L^{1}[0,1], n$ fixed. The solution of the $L^{2}$ version of this problem is a classical result of E. Schmidt [3] (see also Courant and Hilbert [1, p. 161]).

For the class of strictly totally positive kernels $K$, we are able to show that a best choice of functions $u_{1}, \ldots, u_{n}, v_{1}, \ldots, v_{n}$ is determined by certain sections $K\left(x, \xi_{1}\right), \ldots, K\left(x, \xi_{n}\right), K\left(\tau_{1}, y\right), \ldots, K\left(\tau_{n}, y\right)$ of the kernel $K$.

Definition. A real-valued kernel $K(x, y)$, defined and continuous on $[0,1] \times[0,1]$, is called strictly totally positive (S.T.P.) if all its Fredholm minors

$$
K\left(\begin{array}{c}
s_{1}, \ldots, s_{m} \\
t_{1}, \ldots, t_{m}
\end{array}\right)=\operatorname{det}\left\|K\left(s_{i}, t_{j}\right)\right\|_{i, j=1}^{m}
$$

are positive for $0 \leqslant s_{1}<\cdots<s_{m} \leqslant 1,0 \leqslant t_{1}<\cdots<t_{m} \leqslant 1$, and all $m \geqslant 1$.

For every $s=\left(s_{1}, \ldots, s_{m}\right), 0=s_{0}<s_{1}<\cdots<s_{m}<s_{m+1}=1$, define the step function

$$
h_{s}(x)=(-1)^{i}, \quad s_{i} \leqslant x<s_{i+1}, i=0,1, \ldots, m .
$$

Furthermore, let $\|f\|_{1}=\int_{0}^{1}|f(x)| d x$, and

$$
\left(K h_{s}\right)(x)=\int_{0}^{1} K(x, y) h_{s}(y) d y, \quad\left(K^{T} h_{s}\right)(y)=\int_{0}^{1} K(x, y) h_{s}(x) d x .
$$

The following theorem plays a central role in this work.

THEOREM 1. Let $K$ be a S.T.P. kernel. Given $n \geqslant 1$, there exists $\xi=\left(\xi_{1}, \ldots, \xi_{n}\right), 0<\xi_{1}<\cdots<\xi_{n}<1$, such that for any $t=\left(t_{1}, \ldots, t_{n}\right)$, $0<t_{1}<\cdots<t_{n}<1$,

$$
\left\|K h_{\xi}\right\|_{1} \leqslant\left\|K h_{t}\right\|_{1} \text {. }
$$

AMS (MOS) subject classifications (1970). Primary 41 A63, 41 A45.

${ }^{1}$ Sponsored by the United States Army under Contract No. DAAG29-75-C-0024. 
Moreover, $K h_{\xi}$ has exactly $n$ distinct sign changes at $\tau=\left(\tau_{1}, \ldots, \tau_{n}\right), 0<\tau_{1}$ $<\cdots<\tau_{n}<1$, and
(1) $\operatorname{sgn} K h_{\xi}=h_{\tau}$,
(2) $\operatorname{sgn} K^{T} h_{\tau}=h_{\xi}$.

(When $K h_{\xi}$ or $K^{T} h_{\tau}$ are zero in (1) or (2), we assign a value to the sgn so that the equations are valid.)

Corollary. Let $\tau=\left(\tau_{1}, \ldots, \tau_{n}\right)$ be the $\tau$-point defined in Theorem 1 . Then,

$$
\left\|K h_{\xi}\right\|_{1}=\left\|K^{T} h_{\tau}\right\|_{1} \leqslant\left\|K^{T} h_{s}\right\|_{1}
$$

for every s-point, $s=\left(s_{1}, \ldots, s_{n}\right), 0<s_{1}<\cdots<s_{n}<1$.

We are now prepared to state the main theorem. To this end observe that the function

$$
E(x, y)=K\left(\begin{array}{c}
x, \tau_{1}, \ldots, \tau_{n} \\
y, \xi_{1}, \ldots, \xi_{n}
\end{array}\right) / K\left(\begin{array}{c}
\tau_{1}, \ldots, \tau_{n} \\
\xi_{1}, \ldots, \xi_{n}
\end{array}\right)
$$

(where $\xi$ and $\tau$ are obtained from Theorem 1) may be expressed as

where

$$
=K(x, y)-\sum_{i, j=1}^{n} c_{i j} K\left(x, \xi_{i}\right) K\left(\tau_{j}, y\right)
$$

$$
c_{i j}=(-1)^{i+j} K\left(\begin{array}{l}
\tau_{1}, \ldots, \tau_{j-1}, \tau_{j+1}, \ldots, \tau_{n} \\
\xi_{1}, \ldots, \xi_{i-1}, \xi_{i+1}, \ldots, \xi_{n}
\end{array}\right) / K\left(\begin{array}{l}
\tau_{1}, \ldots, \tau_{n} \\
\xi_{1}, \ldots, \xi_{n}
\end{array}\right)
$$

Therefore

$$
\begin{aligned}
E_{1,1}(K) & \equiv \min _{u_{i}, v_{i}} \int_{0}^{1} \int_{0}^{1}\left|K(x, y)-\sum_{i=1}^{n} u_{i}(x) v_{i}(y)\right| d x d y \\
& \leqslant \int_{0}^{1} \int_{0}^{1}|E(x, y)| d x d y .
\end{aligned}
$$

Actually, we have

\section{THEOREM 2.}

$$
\begin{aligned}
E_{1,1}(K) & =\int_{0}^{1} \int_{0}^{1}|E(x, y)| d x d y=\left\|K h_{\xi}\right\|_{1} \\
& =\int_{0}^{1} \int_{0}^{1}\left|K(x, y)-\sum_{i=1}^{n} u_{i}^{0}(x) v_{i}^{0}(y)\right| d x d y,
\end{aligned}
$$

where $u_{i}^{0}(x)=K\left(x, \xi_{i}\right)$, and $v_{i}^{0}(y)=\Sigma_{j=1}^{n} c_{i j} K\left(\tau_{j}, y\right)$. 
Proof. By the Hobby-Rice Theorem [2], we know that given any $n$ functions $v_{1}, \ldots, v_{n} \in L^{1}[0,1]$, there exists a $t=\left(t_{1}, \ldots, t_{k}\right), 0 \leqslant k \leqslant n$, such that $\int_{0}^{1} v_{i}(y) h_{t}(y) d y=0, i=1, \ldots, n$. Let $h(x, y)=h_{t}(y) \operatorname{sgn}\left(K h_{t}\right)(x)$. Then, for any $u_{1}, \ldots, u_{n} \in L^{1}[0,1]$,

$$
\begin{aligned}
\left\|K h_{\xi}\right\|_{1} & \leqslant \int_{0}^{1}\left|\left(K h_{t}\right)(x)\right| d x=\int_{0}^{1} \int_{0}^{1}\left(K(x, y)-\sum_{i=1}^{n} u_{i}(x) v_{i}(y)\right) h(x, y) d x d y \\
& \leqslant \int_{0}^{1} \int_{0}^{1}\left|K(x, y)-\sum_{i=1}^{n} u_{i}(x) v_{i}(y)\right| d x d y .
\end{aligned}
$$

Since $u_{1}, \ldots, u_{n}, v_{1}, \ldots, v_{n}$ were arbitrarily chosen in $L^{1}[0,1]$, we have $\left\|K h_{\xi}\right\|_{1} \leqslant E_{1,1}(K)$. Also, in view of (1), (2), and (3),

$$
\begin{aligned}
\int_{0}^{1} \int_{0}^{1}|E(x, y)| d x d y=\int_{0}^{1} \int_{0}^{1} E(x, y) h_{\tau}(x) h_{\xi}(y) d x d y \\
=\int_{0}^{1}\left(K h_{\xi}\right)(x) h_{\tau}(x) d x-\sum_{i, j=1}^{n} c_{i j}\left(K^{T} h_{\tau}\right)\left(\xi_{i}\right)\left(K h_{\xi}\right)\left(\tau_{j}\right) \\
=\int_{0}^{1}\left|\left(K h_{\xi}\right)(x)\right| d x=\left\|K h_{\xi}\right\|_{1},
\end{aligned}
$$

which, together with (4), finishes the proof.

Full details, extensions, and the relationship of this problem to $n$-widths will appear elsewhere.

\section{REFERENCES}

1. R. Courant and D. Hilbert, Methods of mathematical physics, Vol. 1, Interscience, New York, 1953. MR 16, 426.

2. C. R. Hobby and J. R. Rice, A moment problem in $L_{1}$-approximation, Proc. Amer. Math. Soc. 16 (1965), 665-670. MR 31 \#2550.

3. E. Schmidt, Zur Theorie der Linearen und Nichtlinearen Integralgleichungen. I, Math. Ann. 63 (1907), 433-476.

IBM, T. J. WATSON RESEARCH CENTER, MATH. SCIENCES DEPARTMENT, YORKTOWN HEIGHTS, NEW YORK 10598

MATHEMATICS RESEARCH CENTER, UNIVERSITY OF WISCONSIN, MADISON, WISCONSIN 53706 